the ability of the local healthcare system to provide the required care. Damage to the healthcare infrastructure will further compromise the delivery of health services. Field hospitals (FHs) may provide care for the injured and act as a substitute for destroyed hospitals in the aftermath of sudden-onset disasters. A FH is a large mobile medical unit that temporarily provides care to casualties on-site before they can be transported safely to more permanent hospital facilities. The concept was inherited from the battlefield (such as the mobile army surgical hospital $(\mathrm{MASH})$ ). A FH is defined as a mobile, self-contained, selfsufficient healthcare facility capable of rapid deployment and expansion or contraction to meet immediate emergency requirements for a specified period of time. The FH may be dispatched temporarily with personnel or donated without personnel.

Methods: Field hospitals have been focused on providing emergency trauma care for the initial 48 hours following the suddenonset disasters, but they tend to continue operations much longer after the on-set. The aim of this study was to assess the timing, activities, and capacities of the FHs deployed after a disaster, especially in severe environments, and provide guidelines on the implementation and deployment of a FH during a disaster. In particular, the activity of Italian Field Hospitals utilized during disasters, humanitarian emergencies, and mass-gatherings events will be described.

Conclusions: Field hospitals have been used successfully during disasters, and now have a crucial role in supporting affected populations and damaged healthcare infrastructures. Field hospitals also play a role in the international community response.

Prehosp Disaster Med 2011;26(Suppl. 1):s95-s96

doi:10.1017/S1049023X11003244

\section{(A341) Disaster! What about Me?}

H.N. Al-Qusimy

Accident \& Emergency, Muscat, Oman

Background: There is one important aspect of hospital preparedness for disaster that has so far received minimum attention. It has been taken for granted that medical staff know best how to take care of themselves and are well prepared personally when there is a call for disaster. However, the reality is far from what is expected, and many staff tend to keep their personal needs as a second priority when it comes to patient care, especially during disasters and mass casualties. They may not show it, however, while attending to their duties and managing casualties of disasters.

Discussion: Medical staff continue to have personal concern regarding their family, properties, personal safety and in some instances their own health as well. The medical staff are not the only one to blame, as other parties, including their employers are involved. This presentation will discuss causes and consequences of ill prepared medical staff personnel to disaster or Mass casualty, including a brief illustration of a mini survey that was conducted immediately following a hospital disaster drill. The presentation will also elaborate on developing staff personal response plans and kits that will contribute in reducing the burden of concern for their own family and them and indirectly may help increase their performance and productivity during disaster or mass casualty situations.

Prehosp Disaster Med 2011;26(Suppl. 1):s96

doi:10.1017/S1049023X11003256
(A342) Distribution Network Design in Relief Chain Management: Learnings from the 2008 Kosi Floods, Bihar, India

A. Prakash

Jamsetji Tata Centre for Disaster Management, Mumbai, India

Logistics has always been an important factor in humanitarian aid operations, to the extent that logistics efforts account for $80 \%$ of disaster relief. They often have to be carried out in an environment with destabilized infrastructures ranging from a lack of electricity supplies to limited transport infrastructure. Furthermore, since most natural disasters are unpredictable, the demand for goods in these disasters is also unpredictable. Thus it is evident that humanitarian logistics is challenging as it has to be more flexible, and has to function under severe constraints. In India, humanitarian logistics remains a neglected field ins disaster management, the cost of which is paid by loss of human lives and property. In recent Kosi Flood the total population of $33,45,545$ people living in 993 villages of 412 panchayats of 35 blocks of 5 districts were affected. A total of 3, 40,742 houses were damaged and 7, 12,140 animals were affected. A total of 239 humans and 1232 animal's lives were lost (Department of Planning and Development, Government of Bihar). The Paper analyses, Madhepura district government's mechanisms of managing logistics while responding to Kosi floods 2008. It evaluates the efficiency of these mechanisms with respect to its outreach to flood affected people. It review and analyses the strengths and weaknesses of the adopted distribution network design for relief management with reference to strategic locations of the relief camps, during the first month of the kosi floods in 2008. It suggests ways to improve disaster logistics at district level in Bihar. The study looks into the possibilities of adopting newer approaches in the field of logistics that could be implemented with greater efficiency under similar conditions. The logistics in the devastating floods at the district level in a developing country has key learning lessons for similar resource poor environments.

Prehosp Disaster Med 2011;26(Suppl. 1):s96 doi:10.1017/S1049023X11003268

\section{(A343) Rebuilding Post Conflict Food Security in Liberia} T.W. Graham

N/A, Davis, United States of America

Liberia's 14 year civil war destroyed domestic agricultural production, veterinary and agricultural education, extension services and domestic food security. These losses severely limited domestic food production, and basic hygiene and sanitation: potable water, abattoirs, cold chain and food storage were greatly diminished. The average Liberian life expectancy fell from 45.8 in 1990 to 41.8 years presently. The population birth and death rate are two of the highest globally with a resulting population growth rate, of $2.7 \%$ per annum; this growth rate requires an immediate and concerted focus on domestic food production to alleviate nutritional inadequacy and hunger, trade imbalances and loss of foreign exchange credits. Food supply nationally is presumed adequate because of importation, though domestic production is inadequate. Unequal distribution precludes food security for all Liberians. Value chain augmentation, enhancing 
food availability across all sectors of Liberian society and ensuring distribution of a safe food supply needs critical development. Infant mortality remains one of the highest in the world (approximately 160/1000 births), much of which is attributed to food insecurity, food contamination and lack of uniformly available potable water. Recreation of Liberia's public health and food security requires redevelopment of disease monitoring and laboratory diagnostic capability to re-establish safe food production and handling practices across all sectors. This will allow determination of endemic disease burden for the principal livestock species: poultry, sheep, goats, cattle and swine. Creation of a national disease surveillance/monitoring system allows for targeted disease intervention, ensuring vaccination for correct serotypes and most critically prevalent diseases. Creation of community level training and support will target intervention of local diseases, but also allow for national prioritization of diseases. Targeting which are most prevalent or most likely to cause production limiting effects will require periodic surveillance, targeted vaccination, and chemotherapeutic intervention and evaluation of therapeutic success.

Prehosp Disaster Med 2011;26(Suppl. 1):s96-s97

doi:10.1017/S1049023X1100327X

(A344) Integrating Post-Disaster Reconstruction with Pre-Existing Development Strategies - Experiences from the 2010 Leh Flashflood

\section{A. Prakash, ${ }^{1}$ A. Kumar, ${ }^{1}$ S. Khan ${ }^{2}$}

1. Jamsetji Tata Centre for Disaster Management, Mumbai, India

2. Centre for Development Studies, Mumbai, India

Disasters cause destruction, but also bring opportunities for development. A lack of this perspective in disaster management leads to planning and managerial follies. The reconstruction and rehabilitation program from the Latur earthquake (Maharashtra, India) should be used as a starting point. Leh, the largest district of India, was hit by a cloudburst and subsequent flashfloods on 05-07 August 2010. The monetary value of the total damages is estimated to be approximately Rs 225 crores. A total of $87 \%$ of the affected infrastructure required complete reconstruction. The rest received partial damages. This paper uses field data collected on development needs in 112 villages over a span of five years (just preceding the disaster) and data for damage assessment from the 42 affected villages collected immediately after the disaster. The paper does a careful assessment of the damages in the flashflood, analyses the pre-existing developmental requirements of all the villages in the district, and explores the possibilities of integrating post-disaster reconstruction with the pre-existing developmental requirements. In India, post-disaster reconstruction quite often is an elaboration of short-term, makeshift arrangements, thereby giving rise to obvious developmental concerns. Merits and demerits of this strategy will be discussed. This research examines the financial implications of integrating reconstruction projects with development programs. It also looks into the sustainability of development, and how can this serve as a strategy in making communities more resilient to future disasters.

Prehosp Disaster Med 2011;26(Suppl. 1):s97

doi:10.1017/S1049023X11003281
(A345) The Longitudinal Study of Survivors' Daily Lives and Health Conditions after Sichuan Earthquake in China - The Result of the 3rd Year Compared with the 1st Year and 2nd Year Study

X.Y. Hu, ${ }^{1}$ Y.J. Cheng, ${ }^{1}$ O. Chen, ${ }^{1}$ N. Long, ${ }^{1}$ J.P. Li, ${ }^{1}$

Y.L. Luo, ${ }^{1}$ A. Yamamoto ${ }^{2}$

1. Nursing Department, Sichuan Chengdu, China

2. Research Institute of Nursing Care for People and Community, Hyogo, Japan

Objective: To longitudinally investigates the Survivors' daily lives and healthy condition after Sichuan earthquake in China. This study identifies Survivors' daily lives and health conditions for 3-years.

Ethical Consideration: The study was conducted after obtaining approval in Ethical Review Board of Sichuan University.

Method: The study was conducted for the survivors who experienced the earthquake and aged 18 years and over, and joined the 1 st year and 2nd year study. Questionnaire included the survivors' socio-demographics, impact of disaster and the health status after disaster. The date was analyzed using SPSS 13.0 software.

Results: Results showed the follow-up date which was results of 3-years. The changes of daily lives of survivors for 3-years were fluctuations. As their health conditions in the 3rd year, the following symptoms were decreased: locomotors system disease, respiratory disease, Autoimmunity and endocrine diseases, asthma and allergy, Gout, Irregular menstruation/dysmenorrheal, kidney disease and the others. In contrast, the following symptoms were increased: cardiac disease, weight gain/less, hypertension, diabetes, and liver disease. About their self-rated health, "Not healthy" at 3rd year was $26.4 \%$. Compared with the 1st year and 2nd year results, it has taken a turn for the significantly better (1st year 49.9\%; 2nd year 35.1\%). With regard to the psychological condition, the Positive response of IES-R at 3 rd year was $11.6 \%$. Compared with the 1st year and 2nd year results, the Positive response of IES-R significantly decrease (1st year 49.8; 2nd year 31.8\%. $p<0.001$, by Pearson Chi-square).

Conclusion: After 3rd year of the Sichuan earthquake disaster, the survivors' physical and psychological conditions have improved compared to 1st year and 2nd year results. However most of the survivors still had some health problems in their health. Our local nursing authorities still need to go on their health promotion, which can further improve the survivors' health status.

Prehosp Disaster Med 2011;26(Suppl. 1):s97

doi:10.1017/S1049023X11003293

(A349) The Role of Poison Centers in the 2010 Gulf Oil Spill Response

M. Ryan

Department of Emergency Medicine, Shreveport, United States of America

Introduction: There are 60 Poison Centers in the United States that manage over 2.5 million poison exposure calls each year. A poison center can be reached 24 hours a day by dialing a national 800 hotline. Poison Centers are staffed by Specialists in Poison Information who are highly trained in clinical toxicology and are very skilled in telephone triage. 